\title{
Parroca
}

\section{Paola Manni}

PUBBLICATO: 23 OCTOBER 2020

\section{Quesito:}

E.T. ci chiede come possa essere tradotto in italiano il termine Pfarrerin che nella Chiesa cattolica cristiana svizzera (non romana) indica dell'equivalente di parroco al femminile: come un parroco riceve l'ordinazione presbiterale dal vescovo e l'ufficio è equivalente.

\section{Parroca}

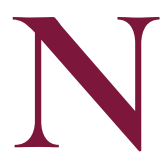

el riproporre il ben noto e dibattuto tema del femminile dei nomi cosiddetti "professionali", la nostra interlocutrice ci pone di fronte a un quesito assai interessante, anche perché inedito entro i nostri confini nazionali, dove siamo al riparo dalla necessità di declinare al femminile parroco, dato che la Chiesa cattolica romana non concede alle donne (almeno per ora) di accedere al sacerdozio. Naturale però che questa necessità si manifesti nell'ambito della Chiesa cattolica cristiana della Svizzera che, a partire dal ${ }_{9999}$, ammette a tale ministero anche le donne. Ed è del tutto lecito che la signora $T$. rivendichi l'utilizzo del femminile parroca e, con solide argomentazioni, rifiuti la traduzione pastora, termine forviante che rimanda alla dottrina protestante, e crea peraltro una diffrazione del tutto illogica rispetto alla corrispondente forma femminile Pfarrerin, già in uso presso i fedeli di lingua tedesca della stessa comunità cattolica cristiana della Svizzera.

Del resto, dal punto di vista grammaticale, la forma parroca non presenta nessun particolare problema, appartenendo alla serie dei sostantivi femminili in -a corrispondenti a maschili uscenti in -o, che già annovera numerosi nomi di mestiere, dai tradizionali maestra e cuoca a quelli di introduzione più recente e ormai acclimatati come sindaca. Parroca è insomma una forma del tutto legittima e tale da non entrare in competizione con possibili alternative, diversamente da quanto accade ad altri termini femminili di ambito religioso che sollecitano qualche ulteriore riflessione. Pensiamo a prete, appartenente alla classe ambigenere in -e e quindi già potenzialmente femminile (si dirà la prete, come si dice la custode, la preside), al quale però la stessa nostra interlocutrice affianca il dotto e inequivocabilmente femminile presbitera; e anche diacona che, per quanto grammaticalmente ineccepibile al pari di parroca, subisce la concorrenza dell'antico diaconessa (dal tardo latino diaconissa a sua volta derivato dal greco diakonissa).

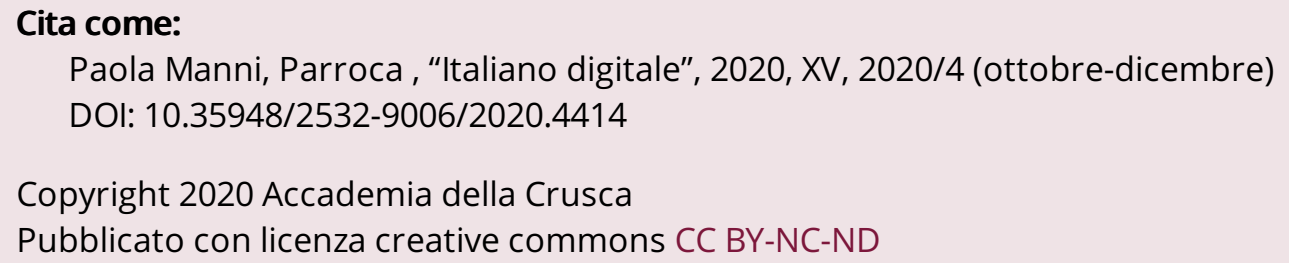

